\title{
Representación de eventos de ruido ambiental a partir de esquemas preconceptuales y buenas prácticas de educción geoespacial de requisitos
}

\author{
Claudia Elena Durango-Vanegas, Paola Andrea Noreña-Cardona, \\ Carlos Mario Zapata-Jaramillo \\ Universidad San Buenaventura, \\ Universidad Nacional de Colombia, \\ Colombia \\ claudia.durango@usbmed.edu.co, \{panorenac,cmzapata\}@unal.edu.co
}

\begin{abstract}
Resumen. El ruido ambiental es un evento que genera contaminación acústica y tiene repercusiones nocivas en la calidad de vida, la salud y el comportamiento humano. Los esquemas preconceptuales son modelos de ingeniería de software para representar diferentes dominios, en los que se suelen modelar eventos. Las representaciones actuales del ruido ambiental se basan en modelos de procesos y estructuras de componentes espaciales para generar mapas de ruido en sistemas de información geográfica (SIG). Sin embargo, les falta considerar la especificación de los eventos y las buenas prácticas de educción geoespacial de requisitos. Estas prácticas permiten relacionar objetos geográficos comunes en SIG. En este artículo se propone una representación de eventos de ruido ambiental basada en esquemas preconceptuales y buenas prácticas de educción geoespacial de requisitos. Esta representación mejora la comprensión de los geodatos requeridos para modelar el comportamiento de los eventos de ruido ambiental en la generación mapas de ruido.
\end{abstract}

Palabras clave: eventos de ruido ambiental, buenas prácticas de educción geoespacial de requisitos, esquemas preconceptuales, ingeniería de software, sistemas de información geográfica.

\section{Environmental Noise Representation by using Pre-conceptual Schemas and Geospatial Elicitation Requirements Best Practices}

\footnotetext{
Abstract. Environmental noise is an event, which generates noise pollution. Such event causes harmful effects in quality of life, health, and human behavior. Preconceptual schemas are software engineering models for representing any domains, in which events are modeled. Current environmental noise 
representations are based on process models and spatial component structures for generating noise maps in geographic information systems (GIS). However, such representations lack events specification and geospatial elicitation requirements good practices. Such practices are used for relating common geographic objects in GIS. In this paper, we propose an environmental noise representation by using pre-conceptual schemas and geospatial elicitation requirements good practices. Such representation improves geodata understanding for modeling environmental noise events behavior in noise maps generation.

Keywords: environmental noise events, geospatial elicitation requirements good Practices, pre-conceptual schemas, software engineering, geographic information systems.

\section{Introducción}

El ruido ambiental es un evento que se considera uno de los principales contaminantes acústicos de la salud en los seres vivos. Por ello, diversas entidades gubernamentales a nivel regional, nacional e internacional realizan estudios de medición del ruido ambiental, buscando mejorar el nivel de calidad de vida y el comportamiento de las personas. Algunas instituciones, como la Organización Mundial de la Salud (OMS), buscan regular el ruido ambiental, por ser la principal causa de preocupación en la salud pública, tratando de encontrar un nivel de confort acústico para las personas afectadas. Con este fin, los eventos de ruido ambiental se miden en una determinada zona de estudio. Los resultados obtenidos indican que el ruido ambiental tiene una alta repercusión nociva en el nivel de calidad de vida, el comportamiento y las actividades cotidianas del ser humano [1].

Los esquemas preconceptuales (EP) son modelos de ingeniería de software para representar un dominio y facilitar la comprensión de los analistas y los interesados. Estos esquemas integran características dinámicas y estructurales que permiten dar una vista completa del dominio en un mismo modelo [2]. Por ello, los EP se utilizan para representar eventos involucrados en cualquier dominio.

Estos eventos permiten analizar el comportamiento del sistema mediante el inicio o el fin de los procesos [3,4]. Aquellos eventos que inician procesos se conocen como eventos disparadores [5]; de esta manera, los eventos de ruido ambiental se clasifican como eventos disparadores.

En algunas representaciones del ruido ambiental se utilizan modelos de procesos como diagramas de flujo [6,7,8], diagramas de clases [9] y diagramas de bloques $[10,11]$, para generar mapas de ruido en sistemas de información geográfica (SIG) a partir de objetos geográficos. Otras representaciones se basan en la integración de estructuras de objetos geográficos para generar mapas de ruido a partir de métodos, tales como colección básica de datos [12], interpolación [13], modelado por escenarios [14] y propagación de sonidos [15]. Los mapas de ruido cobran importancia porque se 
pueden realizar análisis y simulaciones de eventos de ruido ambiental en diferentes lugares y proponer soluciones ante su ocurrencia [16].

A pesar de que las anteriores propuestas incluyen representaciones de eventos de ruido ambiental y algunos objetos geográficos para generar mapas de ruido en SIG, hace falta representar eventos de ruido ambiental. Además, estos trabajos no tienen en cuenta las buenas prácticas de educción geoespacial de requisitos para realizar mapas de ruido.

Estas buenas prácticas permiten identificar un terreno común de las cosas que se deben atender del proyecto SIG para modelar conceptual, física y lógicamente las entidades y fenómenos geográficos requeridos para generar mapas de ruido.

En este artículo se propone una solución a partir de la representación de eventos de ruido ambiental, tomando como base los esquemas preconceptuales. Esta representación integra elementos de las buenas prácticas de la educción geoespacial de requisitos para obtener el catálogo de objetos geográficos, el modelado de las estructuras de geoalmacenamiento y el modelo de georepresentación en los proyectos SIG para generar mapas de ruido.

La representación propuesta mejora la comprensión del proceso de medición de eventos relacionados con ruido ambiental. Además, la definición de las buenas prácticas ayuda a identificar los elementos mínimos que se requieren en la adquisición de geodatos para generar mapas de ruido según zonas específicas de estudio.

La estructura de este artículo es la siguiente: en la sección 2 se presenta el marco conceptual relacionado con los mapas de ruido ambiental, los esquemas preconceptuales y las buenas prácticas de educción geoespacial de requisitos; en la sección 3 se plantea el problema, identificando trabajos similares; en la sección 4 se propone una solución basada en la representación de eventos de ruido ambiental a partir de esquemas preconceptuales y buenas prácticas de educción geoespacial de requisitos; en la sección 5 se aplica la solución, y en la sección 6 se presentan las conclusiones y el trabajo futuro.

\section{Marco conceptual}

\subsection{Eventos de ruido ambiental}

El ruido ambiental es un evento que genera un sonido indeseable que afecta o perjudica a las personas y su entorno, y es una de las principales fuentes de contaminación ambiental en los centros urbanos [13,16]. El crecimiento de la población, la modernización de las actividades cotidianas, el incremento de las industrias y de los medios de transporte son algunas causas del aumento y de la presencia de eventos de ruido ambiental en centros poblados. En los centros urbanos existen diversas fuentes generadoras de contaminación auditiva producto del ruido ambiental, tales como: transporte automotor, construcciones, obras públicas, ruido industrial y ruido propio de establecimientos públicos y de vecindarios. Lo anterior trae como consecuencia un rompimiento del equilibrio natural, generando estrés por el incremento de los niveles permitidos de ruido en las ciudades [17]. Por ello, el ruido 
ambiental se considera un evento de alta repercusión en cambios relacionados con la salud, el comportamiento y las actividades cotidianas de las personas [18]. Los eventos de ruido ambiental se clasifican como eventos disparadores, ya que desencadenan procesos y otros eventos [5].

\subsection{Esquemas preconceptuales}

Los esquemas preconceptuales (EP) son modelos de ingeniería de software para representar cualquier dominio. Los EP integran reglas de la lingüística computacional $\mathrm{y}$ de los modelos conceptuales que permiten un acercamiento al lenguaje natural. Este acercamiento facilita el nivel de comprensión de los analistas y los interesados. Los elementos en la notación de los EP permiten una vista completa del dominio, ya que involucran características dinámicas y estructurales en un mismo modelo [2]. Estos elementos se pueden observar en la Figura 1: relaciones (estructural para las clases, dinámica para los procesos y eventual para los eventos); nodos (condicional, concepto, variable independiente, concepto-clase, y operador); enlaces (implicación, conexión, concepto-nota y operación); y aglutinadores (evento, valor-nota, marco y restricción).

Los EP permiten la representación de eventos disparadores y eventos de resultado (aquellos que son producto de la finalización de los procesos) [5]. Estos eventos permiten el análisis del comportamiento del sistema, ya que ellos cambian los estados de los procesos, por lo que, generalmente, se presentan en la vista dinámica o de comportamiento del sistema $[3,4]$.

\subsection{Buenas prácticas de educción geoespacial de requisitos}

Los sistemas de información geográfica (SIG) son herramientas que permiten adquirir, almacenar, analizar, actualizar y geovisualizar información espacial o geodatos. Los geodatos contienen información espacial y no espacial de un espacio geográfico, asociando la localización y un sistema de coordenadas [19]. Además, son elementos que definen la funcionalidad del SIG y su manipulación permite identificar los productos de trabajo esperados (mapas dinámicos y estáticos). El ciclo de desarrollo de los proyectos SIG involucra la definición de los siguientes elementos: geodatos, geousuarios, software SIG y método SIG.

Las buenas prácticas son procesos aceptados como correctos y efectivos [20]. Por ello, en la literatura se encuentran diversos métodos para planificar proyectos SIG, relacionados con la adquisición, el almacenamiento, el análisis espacial, el mantenimiento, la actualización y la geovisualización de los geodatos. Estos métodos SIG contienen fases, actividades y productos de trabajo que se relacionan con buenas prácticas y en algunos casos se pueden replicar en otros proyectos SIG. Sin embargo, se debe considerar que los proyectos SIG tienen actividades y productos de trabajo propio para su desarrollo y planificación [21], como es el caso de las buenas prácticas de educción geoespacial de requisitos. Estas buenas prácticas se originan en la identificación de prácticas comunes de algunos métodos de desarrollo de proyectos SIG, tales como: la comprensión del problema geoinformático, la identificación de los 
Representación de eventos de ruido ambiental a partir de esquemas preconceptuales y buenas ...

requisitos estructurales de los geodatos y la realización de la estructura de almacenamiento y el catálogo de representación de objetos geográficos.

\section{Planteamiento del problema}

Algunas de las propuestas para la medición y representación del ruido ambiental y la generación de mapas de ruido incluyen modelos de procesos, como diagramas de flujo, a partir del proceso de mapeo de ruido tradicional, para representar las fases de medida de los datos [6]; otras propuestas utilizan este mismo diagrama para calcular el mapa de ruido con base en las mediciones de los datos y sus procesos [7,8]. El diagrama de clases [9] se propone para representar objetos geográficos del ruido ambiental a partir de la vista estructural del proyecto SIG; sin embargo, esta representación carece de elementos para representar eventos y las buenas prácticas de educción geoespacial de requisitos. Otras propuestas utilizan los diagramas de bloques [10,11] como modelos de procesos. Estos modelos tienen una vista dinámica del SIG y se utilizan para representar la secuencia y medida de los geodatos en la generación de mapas de ruido.

Otras propuestas utilizan representaciones que se basan en la integración de estructuras de objetos geográficos a partir de métodos como colección básica de datos [12] para generar mapas de ruido ambiental del tráfico de áreas urbanas.

La interpolación [13] también es un método para generar mapas de ruido ambiental a partir de la predicción de valores para generar mapas de ruido. El modelado por escenarios [14] y la propagación de sonidos [15] son métodos en los que se suelen representar los datos que surgen en el mapa de ruido.

Las anteriores propuestas incluyen algunos métodos y objetos geográficos para generar mapas de ruido; sin embargo, estas propuestas carecen de la representación de eventos de ruido ambiental y los eventos que intervienen en el proceso de medición del ruido. Adicionalmente, no se relacionan los procesos con los objetos geográficos para una representación completa de los elementos y relaciones, que permitan el análisis de la información. Tampoco se encuentra una integración del conjunto de buenas prácticas de educción geoespacial de requisitos que complementen los elementos y la información de los sistemas de información geográfica para la generación de mapas de ruido.

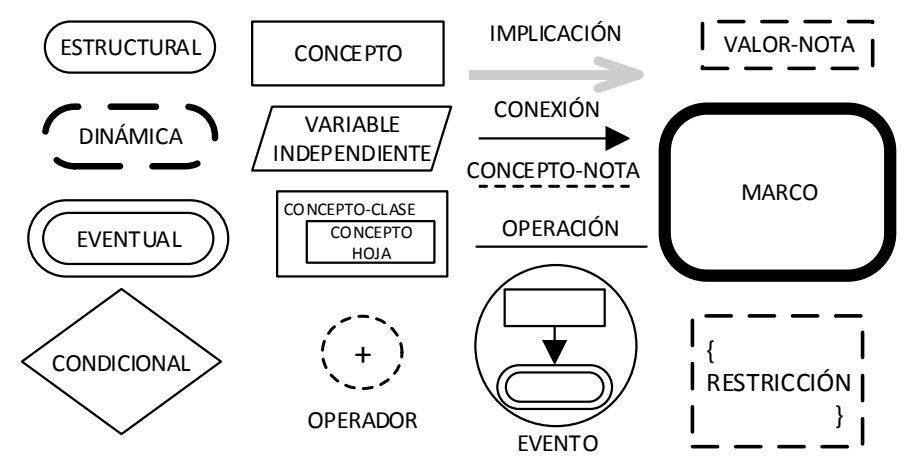

Fig. 1. Notación de los EP. 


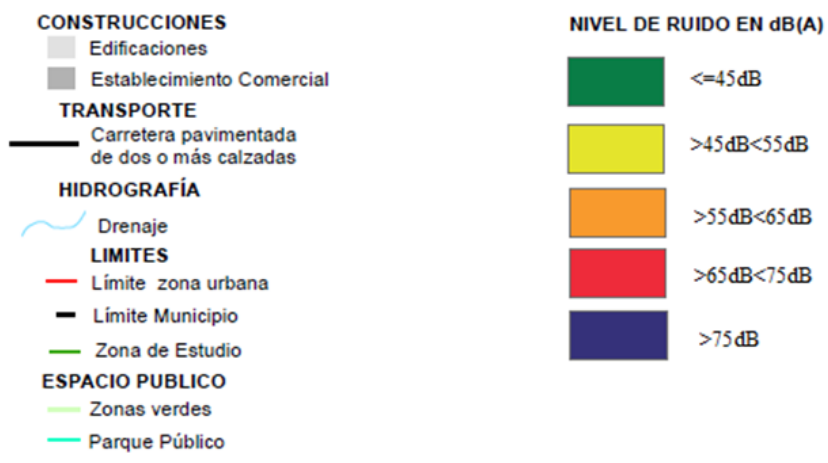

Fig. 2. Catálogo de representación de los objetos geográficos.

\section{Propuesta de solución}

\subsection{Definición de actividades en las buenas prácticas de educción geoespacial de requisitos}

En esta primera etapa se proponen las siguientes actividades relacionadas con las buenas prácticas de educción geoespacial de requisitos para la representación de eventos de ruido ambiental:

Catálogo de objetos geográficos. Identificar los requisitos estructurales de los geodatos. En esta actividad se identifican las entidades, atributos, dominios, relaciones, sistema de coordenadas, escala y límites geográficos, entre otros, para conformar el catálogo de objetos geográficos que debe contener la medición del ruido ambiental. Esta información se obtiene a partir de la interacción entre un geousuario experto en georreferenciación (especialista SIG) y el analista de geodatos; algunos objetos geográficos son zona (zona de interés donde se realiza la medición de los eventos de ruido ambiental), uso del suelo (conjunto de edificaciones en la misma zona) y edificación (espacio en el cual se presenta una construcción). Como información de la edificación se definen el área, el perímetro, la altura y la cantidad de pisos. Estos objetos y su información se deben incluir en la representación de eventos de ruido ambiental. Además, esta información se vincula con el proyecto SIG mediante los programas ArcGIS ${ }^{\circledR}$ para la creación de los mapas de ruido y SoundPLAN® para el análisis de resultados, por lo cual se deben integrar los diferentes elementos y deben ser de fácil comprensión.

Catálogo de representación de objetos geográficos. Realizar la estructura de georepresentación de los geodatos. En esta actividad se identifican los símbolos y los patrones de colores asociados con los niveles de ruido ambiental presentes en los mapas de ruido, como se puede observar en la Figura 2. Para el caso de los niveles de ruido, la especificación de colores constituye una escala de valores, tales como: $<=45 \mathrm{~dB}$ constituye una alerta verde, $>45 \mathrm{~dB}<55 \mathrm{~dB}$ indica una alerta amarilla, $>55 \mathrm{~dB}<65 \mathrm{~dB}$ 
Representación de eventos de ruido ambiental a partir de esquemas preconceptuales y buenas ...

indica una alerta naranja, $>65 \mathrm{~dB}<75 \mathrm{~dB}$ indica una alerta roja $\mathrm{y}>75 \mathrm{~dB}$ indica el mayor valor con una alerta azul.

Modelado de geoalmacenamiento. Realizar la estructuración de almacenamiento de los geodatos. En esta actividad se modelan los geodatos requeridos según el catálogo de objetos geográficos, buscando mejorar la comprensión de las estructuras de geoalmacenamiento requeridas en los proyectos SIG en la generación de mapas de ruido, mediante el modelado de geoalmacenamiento de las estructuras geográficas requeridas para la generación de los mapas de ruido.

\subsection{Representación de eventos de ruido ambiental a partir de esquemas preconceptuales y buenas prácticas de educción geoespacial de requisitos}

En esta segunda etapa se utilizan los EP como propuesta de solución, para facilitar la comprensión de la información entre los geousuarios expertos en georreferenciación y los analistas de geodatos. Por lo tanto, se elabora el esquema preconceptual de la Figura 3 para integrar actividades de las buenas prácticas de educción geoespacial de requisitos que se definen en la Sección 4.2. Este EP involucra los geodatos, los procesos y los eventos que intervienen en la representación de eventos de ruido ambiental para dominios de software científico, que se basan en información geográfica como gestión ambiental. El cumplimiento de esta etapa permite identificar los geodatos que se requieren para generar mapas de ruido según zonas específicas de estudio, en los cuales se centra el modelo.

Para la integración de la actividad de catálogo de objetos geográficos se utilizan las características estructurales de los EP, que permiten observar las relaciones entre conceptos clase y conceptos hoja o atributos mediante la relación estructural tiene. De esta manera, los objetos geográficos o geodatos zona, edificación, uso del suelo, y ruido ambiental son conceptos-clase. A estos conceptos se suman ingeniero de sonido y medición como elementos que complementan la representación del dominio en eventos de ruido ambiental. Zona tiene dos conceptos hoja código y nombre. Zona se relaciona estructuralmente con edificación, uso del suelo y medición. Edificación tiene código, área, altura de pisos, altura, cantidad de pisos, perímetro, cantidad de ocupantes y cantidad de viviendas. Uso del suelo tiene código y nombre. Medición tiene los conceptos hoja código, fecha, cantidad de registros, suma de valores, valor promedio $\mathrm{y}$ alerta final. Ingeniero de sonido tiene identificación y nombre y se relaciona estructuralmente con medición. Ruido ambiental es un concepto-clase que se deriva de los registros que se toman desde un sonómetro y tiene registro, tiempo local, valor y alerta (verde, amarilla, naranja, roja y azul). Los conceptos hoja que se mencionan permiten representar la información de los sistemas de información geográfica que se requieren en la generación de mapas de ruido y en el geoalmacenamiento.

Las características dinámicas de los EP permiten analizar el flujo de los procesos que interactúan con los objetos geográficos mediante relaciones dinámicas y eventuales. Las relaciones dinámicas son operaciones que realiza un objeto animado (persona o rol) como ingeniero de sonido actualiza medición y las relaciones eventuales son operaciones automáticas que se realizan sin la intervención de un objeto animado como ruido ambiental aparece, por lo que pueden incluir relaciones dinámicas sin 
objeto animado en sus restricciones. Para el inicio del flujo se requiere conocer las condiciones iniciales del dominio, las cuales se representan mediante una especificación que incluye los valores iniciales de los parámetros (constantes) y las variables independientes o globales. El flujo inicia a partir del evento disparador tiempo pasa que tiene como restricción incrementar en '1.00' hora digital, mientras se cumpla la condición término <24; este término permite la ubicación del vector tiempo[término] incrementando de 1 en 1 hasta 24 como representación de 1 día, con condiciones iniciales término $=0$ y tiempo[término] $=0.00$.

Durante el tiempo el ruido ambiental aparece, este evento tiene una restricción que permite representar la probabilidad de ocurrencia del ruido ambiental, mediante la relación dinámica automática actualiza aleatorio, la cual es igual al operador rand (función random o aleatorio), esta representación se utiliza con base en eventos aleatorios de probabilidad estadística que utilizan generadores de valores aleatorios en procesos de simulación de sistemas, ya que la ocurrencia del ruido ambiental es variable. Si surge un valor que cumpla la condición aleatorio $>=$ umbral (parámetro que es igual a ' 0,7 ' según las condiciones iniciales, el valor del umbral se estima con base en el conocimiento histórico del proceso) entonces se "activa" la ocurrencia con la ejecución de la relación dinámica actualiza ocurrencia que tenía previamente como condición inicial el valor de "inactiva", si no se cumple la condición tendrá este mismo valor inicial. El evento ruido ambiental aparece implica el evento medición llega, el cual tiene como restricción la condición si tiempo[término]>=medición tiempo inicial y si tiempo[término]<=medición tiempo final entonces inserta el tiempo local y el valor del ruido ambiental.

En la integración de la actividad de catálogo de representación de objetos geográficos_se incluyen los colores de las alertas mediante el uso del evento alerta de ruido ambiental emerge, que se dispara con el evento medición llega. Este evento contiene una restricción que incluye cinco condiciones que representan los niveles de ruido ambiental, los cuales se comparan con el valor del ruido ambiental que se insertó previamente: si el valor del ruido ambiental es $<=45 \mathrm{~dB}$ inserta alerta del ruido ambiental "verde", sino si el valor del ruido ambiental es $>45 \mathrm{~dB}<55 \mathrm{~dB}$ actualiza alerta del ruido ambiental a "amarilla", sino si el valor es $>55 d B<65 d B$ actualiza alerta del ruido ambiental a "naranja", sino si el valor es $>65 \mathrm{~dB}<75 \mathrm{~dB}$ actualiza alerta del ruido ambiental a "roja" sino si el valor es $>75$ actualiza alerta del ruido ambiental a "azul".

El evento medición llega implica la relación dinámica ingeniero de sonido gestiona (inserta, actualiza, elimina y consulta) medición, al igual que el evento condicional si tiempo[término]>=medición tiempo final. Esto quiere decir, que el ingeniero de sonido debe esperar a que el sonómetro termine de registrar los valores, para gestionar la información. Cuando se cumple la restricción del evento medición llega y el evento condicional, el ingeniero de sonido ingresa la información de la medición (código, fecha, código de zona, identificación del ingeniero, cantidad de registros, suma de valores, valor promedio y alerta final). El valor promedio de la medición surge como atributo derivado de la suma de valores y la cantidad de registros, es decir, se calcula al ingresar ambos valores. Al ingresar el registro, el ingeniero también podrá actualizar, eliminar y consultar la información de la medición. 
Representación de eventos de ruido ambiental a partir de esquemas preconceptuales y buenas ...

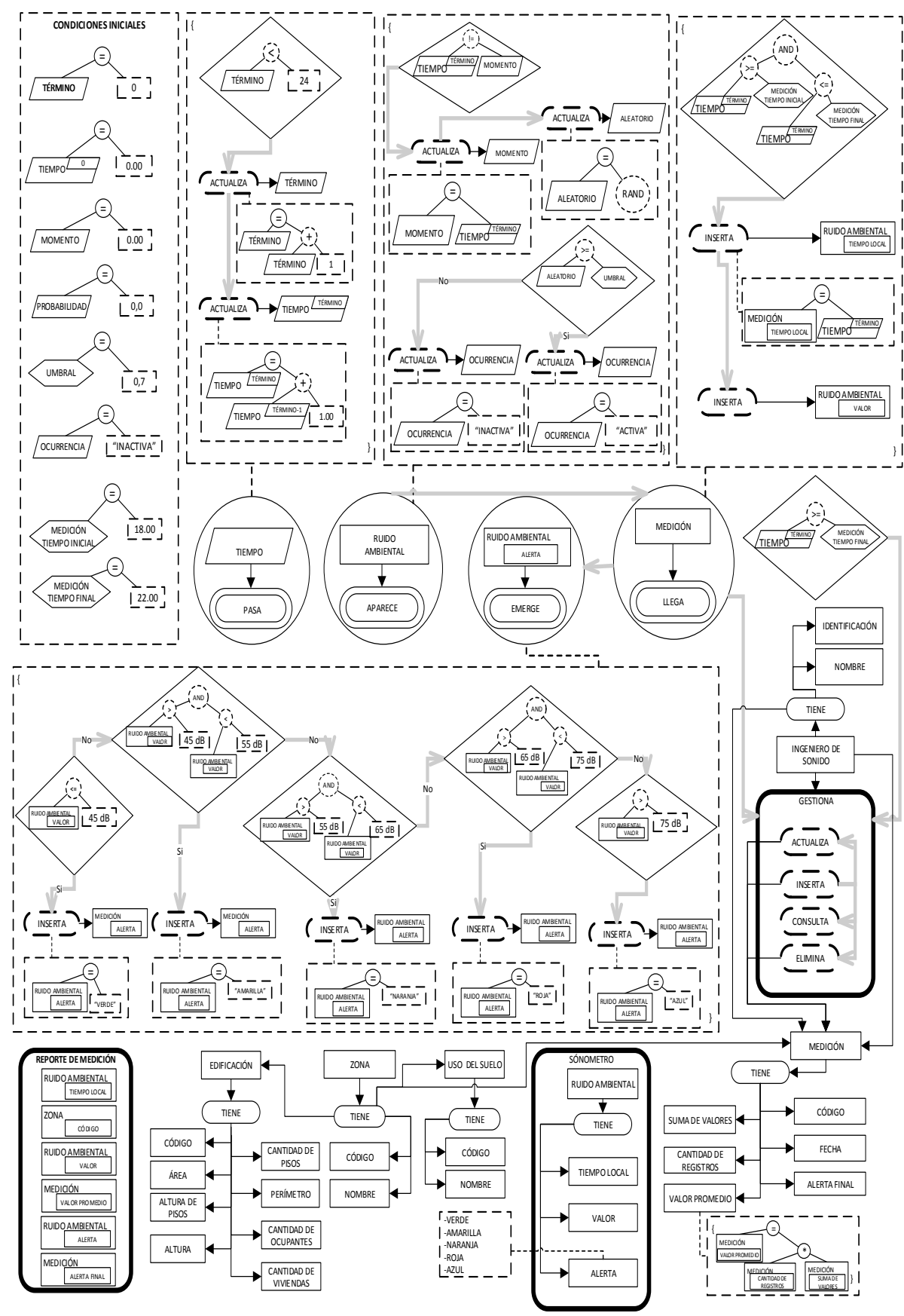

Fig. 3. Representación de eventos de ruido ambiental a partir de EP y buenas prácticas de educción geoespacial de requisitos. 
La integración de la actividad de modelado de geoalmacenamiento se realiza a partir de las características estructurales de los EP y los eventos, en los cuales se puede observar la información de la representación mediante tablas de geoalmacenamiento que representan la base de datos geoespacial de los sistemas de información geográfica y el funcionamiento de variables independientes utilizando tablas de geodatos.

\section{Aplicación}

Las tablas de la base de datos geoespacial que se integran en la actividad de modelado de geoalmacenamiento permiten la aplicación de los geodatos, clases y eventos del esquema preconceptual de la Figura 3, los conceptos hoja de los geodatos en las tablas permiten guardar los valores que corresponden a cada concepto. El caso de aplicación se realiza en la zona de la Comuna 11 Laureles-Estadio de la ciudad de Medellín-Colombia, según el polígono (entidad utilizada para representar superficies) de la Figura 4.

Para iniciar el proceso los ciudadanos que sienten perjuicio en los niveles de ruido pueden llamar a la Subdirección Ambiental del Área Metropolitana para solicitar el servicio de verificación de ruido, según la Resolución 0627 por regulación del Ministerio de Ambiente, Vivienda y Desarrollo Territorial 2006 [22].

El ingeniero de sonido lleva el sonómetro a la zona para medir los niveles de ruido ambiental y programa el tiempo inicial y final (parámetros del EP de la Figura 3) en el sonómetro. La simulación del tiempo se define para un día, pero se puede modificar según las condiciones de otras zonas y lugares de un país. Por ello, la Tabla 1 presenta el funcionamiento de la variable independiente tiempo y la Tabla 2 de las variables independientes probabilidad y ocurrencia (con valores aleatorios que se definen en la ocurrencia de un evento estadístico, permiten indicar cuando se activa el evento de ruido ambiental), las cuales no se almacena en la base de datos geoespacial.

Por su parte, las Tablas 3 a 8 se registran como datos de geoalmacenamiento, ya que el concepto-clase ingeniero de sonido en la Tabla 3 permite registrar quién está a cargo de la medición.

En la Tabla 4 se registran los geodatos de la zona de estudio de la Comuna 11 Laureles-Estadio, para el caso de zona con código "Z701234". En la Tabla 5 se registran los geodatos de edificaciones que pertenecen a la zona, el código "1110001" de edificación corresponde a un Hotel y el "1111012" corresponde a una zona Mixta (residencial y discotecas; véase la Figura 4); Hotel, Mixta y residencial (edificios residenciales) son usos del suelo en la Tabla 6.

La Tabla 7 del geodato ruido ambiental se llena automáticamente mediante el sonómetro tomando cinco registros desde la medición inicial hasta la final (estos registros generalmente se toman en una memoria USB y se llevan a la base de datos geoespacial), los cuales varían entre "74dB" y "77dB". Finalmente, la Tabla 8 permite insertar los datos de la medición como la cantidad de registros " 5 ", el valor promedio "76dB" y la alerta final "Azul", que indica la máxima alerta. En la Figura 5 se observa el mapa de ruido de la zona de estudio con los datos del geoalmacenamiento. 


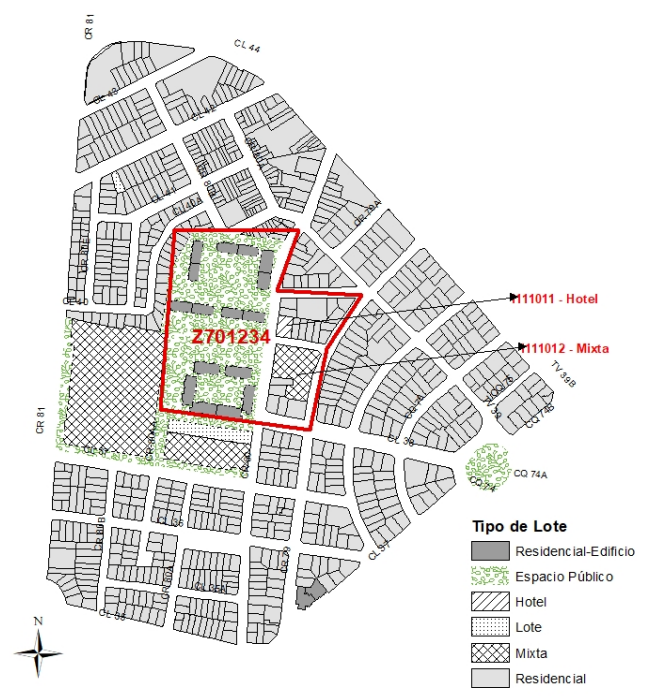

Fig. 4. Zona de estudio "Z701234" Carrera 80 entre Calle 38 y 40. Laureles de Medellín- Colombia.

Tabla 1. Funcionamiento de la variable independiente tiempo.

\begin{tabular}{cc}
\hline \multicolumn{2}{c}{ Tiempo Pasa } \\
\hline Término & Tiempo \\
\hline 0 & 0.00 \\
\hline 20 & 20.00 \\
\hline 21 & 21.00 \\
\hline 22 & 22.00 \\
\hline
\end{tabular}

Tabla 2. Funcionamiento de las variables independientes probabilidad y ocurrencia.

\begin{tabular}{ccc}
\hline & Ruido Ambiental Aparece & \\
\hline Tiempo & Probabilidad & Ocurrencia \\
\hline 0.00 & 0,0 & INACTIVA \\
\hline$\ldots 18.00$ & 0,7 & ACTIVA \\
\hline 19.00 & 0,8 & ACTIVA \\
\hline
\end{tabular}

Tabla 3. Tabla del concepto-clase ingeniero de sonido.

\begin{tabular}{ccc}
\hline \multicolumn{2}{c}{ Ingeniero De Sonido } \\
\hline Código & Nombre \\
\hline 1893213 & Jonathan Ochoa \\
\hline
\end{tabular}


Tabla 4. Tabla del geodato zona.

\begin{tabular}{cc}
\hline \multicolumn{2}{l}{ Zona } \\
\hline Código & Nombre \\
\hline Z701234 & Carrera 80 Entre Calle 38 Y 40 \\
\hline
\end{tabular}

Tabla 5. Tabla del geodato edificación.

\begin{tabular}{cccccccc}
\hline \multicolumn{7}{c}{ EDIFICACIÓN } \\
\hline Cód. & Área & Perímetro & Altura & $\begin{array}{c}\text { Altura } \\
\text { Pisos }\end{array}$ & $\begin{array}{c}\text { Cantidad } \\
\text { Pisos }\end{array}$ & $\begin{array}{c}\text { Cantidad } \\
\text { Viviendas }\end{array}$ & $\begin{array}{c}\text { Código } \\
\text { Zona }\end{array}$ \\
\hline 1111011 & $629,3 \mathrm{~m}^{2}$ & $102,0 \mathrm{~m}$ & $5 \mathrm{~m}$ & $2,5 \mathrm{~m}$ & 2 & 10 & Z701234 \\
\hline 1111012 & $3609,3 \mathrm{~m}^{2}$ & $279,1 \mathrm{~m}$ & $5 \mathrm{~m}$ & $2,5 \mathrm{~m}$ & 2 & 5 & Z701234 \\
\hline
\end{tabular}

Tabla 6. Tabla del geodato uso de suelo.

\begin{tabular}{ccc}
\hline & USO DE SUELO & \\
\hline Cód. & Nombre & Zona \\
\hline U1 & Hotel & Z701234 \\
\hline U2 & Mixta & Z701234 \\
\hline U3 & Residencial & Z701234
\end{tabular}

Tabla 7. Tabla del geodato ruido ambiental.

\begin{tabular}{ccl}
\hline & RUIDO AMBIENTAL & \\
\hline Tiempo Local & Valor & Alerta \\
\hline 18.00 & $74 \mathrm{~dB}$ & Roja \\
\hline 19.00 & $75 \mathrm{~dB}$ & Roja \\
\hline 20.00 & $77 \mathrm{~dB}$ & Azul \\
\hline 21.00 & $76 \mathrm{~dB}$ & Azul \\
\hline 22.00 & $77 \mathrm{~dB}$ & Azul \\
\hline
\end{tabular}

Tabla 8. Tabla de datos del concepto-clase medición.

\begin{tabular}{cccccccc}
\hline \multicolumn{7}{c}{ MEDICIÓN } \\
\hline \multirow{2}{*}{ Cód. } & \multirow{2}{*}{ Fecha } & $\begin{array}{c}\text { Id } \\
\text { Ingeniero }\end{array}$ & $\begin{array}{c}\text { Cód } \\
\text { Zona }\end{array}$ & $\begin{array}{c}\text { Suma } \\
\text { Valores }\end{array}$ & $\begin{array}{c}\text { Cantidad } \\
\text { Registros }\end{array}$ & $\begin{array}{c}\text { Valor } \\
\text { Promedio }\end{array}$ & $\begin{array}{c}\text { Alerta } \\
\text { Final }\end{array}$ \\
\hline \multirow{2}{*}{ M4031004 } & $5 / 03 / 2018$ & 1893213 & Z70234 & $379 \mathrm{~dB}$ & 5 & $76 \mathrm{~dB}$ & Azul \\
\hline
\end{tabular}

\section{Conclusiones y trabajo futuro}

La definición de las buenas prácticas ayuda a identificar los elementos que se requieren en la abstracción de geodatos para representar eventos de ruido ambiental y generar mapas de ruido según zonas específicas de estudio en las fases de educción de requisitos y de planeación y diseño de proyectos SIG.

Para la representación de eventos de ruido ambiental se toman como base los esquemas preconceptuales para generar el catálogo de objetos geográficos, el modelo 


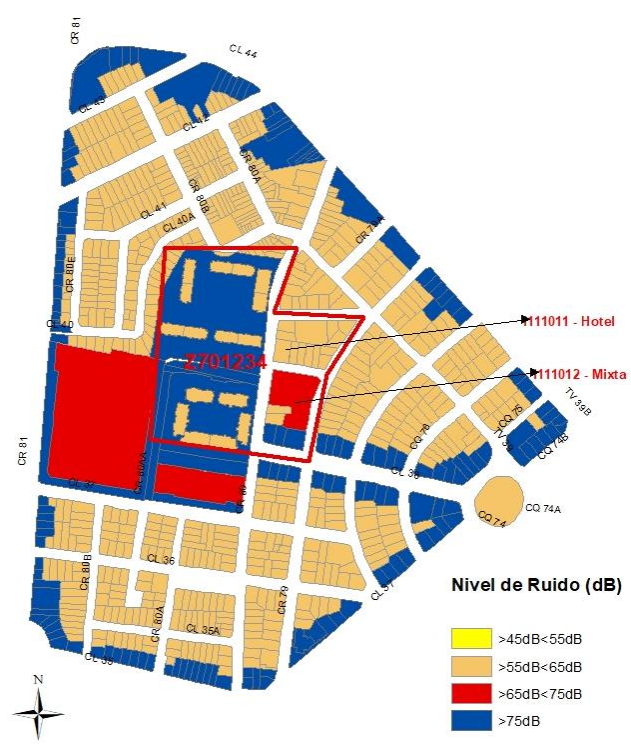

Fig. 5. Mapa de Ruido de la zona de estudio.

de geoalmacenamiento y el catálogo de representación de objetos geográficos según las buenas prácticas de educción geoespacial de requisitos en los proyectos SIG para generar mapas de ruido.

La representación propuesta ayuda a la comprensión del proceso de medición de eventos relacionados con ruido ambiental, ya que presenta los objetos geográficos, los procesos y eventos que interactúan en el proceso.

Como trabajo futuro se pueden representar eventos naturales a partir de modelos matemáticos que ayuden a simular los eventos desde dominios de software científico para prevenir riesgos y desastres.

Agradecimientos. Este artículo es producto del proyecto de investigación Doctoral: Una extensión a esquemas preconceptuales para el refinamiento en la representación de eventos y la notación matemática, con código Hermes 39886, de la Universidad Nacional de Colombia, que financia Colciencias en la convocatoria 727 de becas para estudiantes de doctorado en Colombia. Además, a la Universidad de San Buenaventura, Medellín, por apoyar el proyecto de investigación Doctoral: Definición de buenas prácticas de desarrollo de Sistemas de Información Geográfica utilizando el núcleo de Semat, con el proyecto de investigación "Competencias Semat para un equipo de desarrollo de Proyectos SIG”, con código M4832.

\section{Referencias}

1. Bejarano, J. S.: Gestión del ruido ambiental en Valencia. Modelling in Science Education and Learning, 11(1), pp. 25-42 (2018) 
2. Zapata, C.M.: The UNC-Method revisited: elements of the new approach. Saarbrucken: Lambert (2012)

3. Zapata, C.M., Noreña, P.A., Vargas, F.A.: The Event Interaction Game: Understanding Events in the Software Development Context. Development in Business Simulation and Experiential Learning, 41, pp. 256-263 (2014)

4. Noreña, P.A., Zapata, C.M.: A Game for Learning Event-Driven Architecture: Preconceptual-Schema-based Pedagogical Strategy. Development in Business Simulation and Experiential Learning, 45, pp. 312-319 (2018)

5. OMG, Object Management Group: Business Process Model and Notation BPMN. Standard Document. http://www.omg.org/spec/BPMN/1.2 (2016)

6. Murphy, E., King, E.A.: Smartphone-based noise mapping: integrating sound level meter app data into the strategic noise mapping process. Science of The Total Environment, 562, pp. 852-859 (2016)

7. Wei, W., Van, T., De Coensel, B., Botteldooren, D.: Dynamic noise mapping: A map-based interpolation between noise measurements with high temporal resolution. Applied Acoustics, 101, pp. 127-140 (2016)

8. Li, N., Feng, T., Wu, R.: Flexible distributed heterogeneous computing in traffic noise mapping, in Computers. Environment and Urban Systems, 65, pp. 1-14 (2017)

9. Herman, L., ̌̌ezník, T.: Web 3D visualization of noise mapping for extended INSPIRE buildings model. In: International Symposium on Environmental Software Systems, Neusiedl am See (2013)

10. Rao, A., Han, W.: An Adaptive Gaussian Particle Filter based Simultaneous Localization and Mapping with dynamic process model noise bias compensation. In: IEEE 7th International Conference on Cybernetics and Intelligent Systems (CIS), IEEE Conference on Robotics, Automation and Mechatronics (RAM), Siem Reap, Cambodia (2015)

11. Socoró, J.C., Ribera, G., Sevillano, X., Alías, F.: Development of an Anomalous Noise Event Detection Algorithm for dynamic road traffic noise mapping. In: Proceedings of the 22nd International Congress on Sound and Vibration (ICSV22) (2015)

12. Cai, M., Zou, J., Xie, J., Ma, X.: Road traffic noise mapping in Guangzhou using GIS and GPS, in Applied Acoustics, 87, pp. 94-102 (2015)

13. Gómez, D.: Resolución espacial en la elaboración de mapas de ruido por interpolación. Ingenierías USBMed, 8(1), pp. 56-62 (2017)

14. Suárez, E., Barros, J. L.: Traffic noise mapping of the city of Santiago de Chile. Science of the total environment, 466, pp. 539-546 (2014)

15. Bozkurt, T.S., Demirkale, S. Y.: The field study and numerical simulation of industrial noise mapping. Journal of Building Engineering, 9, pp. 60-75 (2017)

16. Zhao, J., Qin, Q., Xie, C., Wang, J., Meng, Q.: An efficient method of predicting traffic noise using GIS. In: IEEE International Geoscience and Remote Sensing Symposium (IGARSS) (2013)

17. Bajarano, J., Diago, S.: Gestión del ruido ambiental en Valencia. Modelling in Science Education and Learning, 11(1), pp. 25-42 (2018)

18. Cohen, A.M., Salinas, O.: Contaminación auditiva y ciudad caminable. In: Estudios demográficos y urbanos, 32(94), pp. 65-96 (2017)

19. Durango, C.: Asociación de datos espacio-temporales en bases de datos Oracle. Ingenierías USBMed, 5(2), pp. 100-108 (2014) 
Representación de eventos de ruido ambiental a partir de esquemas preconceptuales y buenas ...

20. Torres, D.M., Zapata, C.M.: Best practices of interoperability among heterogeneous software systems: a Semat-based representation. Revista Facultad de Ingeniería, 26(44), pp. 155 (2017)

21. Durango C.E., Zapata, C.M.: Una representación basada en Semat y RUP para el Método de Desarrollo SIG del Instituto Geográfico Agustín Codazzi. Ingenierías USBMed, 6(1), pp. 24-37 (2015)

22. Casas, O., Betancur, C.M., Montaño, J.S.: Revisión de la normatividad para el ruido acústico en Colombia y su aplicación. Entramado, 11(1), pp. 264-286 (2015) 